\title{
Giese, Rachel. (2018). Boys: What it Means to Become a Man. Toronto: Patrick Crean Editions.
}

\author{
Reviewed by: Daniel Quarrie, MacEwan University
}

"Boys: What it Means to Become a Man" is a book written by Rachel Giese. It is a semiautobiography that goes into the various factors that affect boys while drawing on real-world events. Giese analyzes many aspects of what constitutes or makes a boy, in the eyes of society, a man. The four main areas that distinguish a boy from anything else in society are: (1) the Man Box; (2) perception of gender roles; (3) genetic differences (between males and females); and (4) inadequate sexual education.

"The Man Box" was an abstract concept used to describe anything that could only be associated with being a man. The man box typically uses words such as stud, strong, and emotionless (p. 1). The making of "The Man Box" was simple; there just had to be a denial of everything that was perceived as soft, gentle, or feminine (p. 2). To be placed in the man box, the person must be male and fervently so. Any hint of being feminine or appearing soft would be met by ridicule and hostility (p. 4). Males that are typically found in the man box have internalized certain masculine character traits. Promundo (a global non-profit organization) depicts people as "being in the Man Box," if they deeply internalized: "being self-sufficient, acting tough, being physically attractive, sticking to traditional and rigid gender roles, being heterosexual, having sexual prowess, and using aggression to resolve conflicts" (p. 11). These seven traits were called 
the seven "pillars of masculinity," and if a male had deeply internalized these ideas, then they were most likely in "The Man Box" (p. 11).

A boy's reason to be a part of the man box is because of the changing social situations that are creating massive changes in the world (such as \#MeToo) (p. 9). Deeply troubled by how fast the world is changing and evolving, some boys double down on the traits that comprise the man box to have a sense of surety and purpose (p. 13). Internalizing these traits, however, does come with its fair share of drawbacks. Boys found in the man box are at a higher risk of putting their health and safety in danger, being involved in violent crime, or sexually harass women (p. 14).

Boys learn to internalize gender roles from an early age. From elementary school, boys have learned to differentiate what society has attributed to the different sexes but more specifically for their male sex (p. 2). Gender roles for boys and "The Man Box" do share similarities which include "being the head of the household" and being "emotionless" (p. 1). Showing emotion is associated with being feminine, so to eradicate this trait, boys are often told to "tough it out" or "man up" because it is not masculine, and it is the job of the woman to be emotional and sensitive and not the male's (p. 16). Boys learn to kill certain parts of their personalities to adhere to these gender roles that have been placed onto them by society (p. 16).

Gender differences between males and females can be quite speculatory and full of false information. Giese analyzed the hogwash as well as the facts about genetic differences between males and females. Giese mentions that theories that are made to divide the two sexes are propelled by new inventions such as positron emission tomography (PET) scans and functional magnetic resonance imagining (fMRI), which deals with the brain (p. 36). These new inventions give people, 
some psychologists, leeway to provide unreliable generalizations about the differences between males and females. Giese, however, does agree that there is indeed a difference between males and females. There is a difference in brain size, with males having a larger brain on average, and she also cited that the brains of the two sexes develop at different rates in select areas (p. 37). In the realm of learning disabilities, more boys are diagnosed with learning disabilities than girls, which include attention deficit disorder and autism spectrum disorder (p. 20 and 37).

Statistically, boys are being born more often than girls globally, at a rate of 105 boys to 100 girls (p. 27). Giese noted that humans have almost always preferred boys, and even now, families would keep having children until they had a boy, "the true genetic prize" (p. 27). The only situation where it seems girls are consistently preferred over boys is adoption. Parents opt for girls more than boys in this situation because girls appear more vulnerable while boys may bring trouble into their family (p. 28).

Sexual education in North America has not been the best for boys. While sexual education should be beneficial for both sexes, girls are most benefited by the subject matter, and heterosexual males are most ignored when it comes to the subject (p. 167). The majority of sexual education in North America is comprised of abstinence programs and the disaster model approach (p. 173). Abstinence-only sexual education is not very effective for young teenagers with hormonal changes and developments (p. 173). These programs are mainly the only form of sexual education found in America's public schools (p. 176). On the other hand, the disaster model focuses on the negatives of sex like sexually transmitted diseases (STDs) and unwanted pregnancies (p. 173). This approach teaches children to avoid sex because it is dangerous and leads to terrible consequences. "They 
presume sexual activity and sexual violence," and do not try to build emotional health, communication, and healthy, loving relationships (p. 177).

Since sexual education is inadequate, boys and girls turn to porn to learn about sex (p. 179). Boys have a more positive outlook of sex while girls do not (p. 179). The problem with children (young boys in particular since they enjoy it more) learning about sex from porn is that it is unrealistic as to how sex should be performed and how the actual act will progress with a partner (p. 179).

Differences in sexual education led to different experiences in sex for teenagers. Many Dutch teenagers thought their first sexual experiences were "well-timed, wanted, and fun" compared to American teenagers who wished they had waited a bit longer to lose their virginity (p. 181). This stems from the comprehensive sexual and relationship education that is in the Netherlands (p. 180). Teenagers in the Netherlands spend a longer time learning about the intimateness of a relationship and how to respect the other person in the relationship while also learning about stereotypes that plague sex (p. 181). Cultural differences in intimacy have impacted American boys in their experiences in sex (p. 183). All boys crave closeness, but American (also Canadian and British) boys are expected to be emotionally invulnerable, which lessens their intimacy levels and makes them less equipped to handle and be confident in their romantic relationships (p. 183).

Giese concludes by stating that the comparison between male and female is being focused on too keenly (p. 195). The actual situation is not a "zero-sum game" in which one sex has to beat 
the other (p. 195). In order to move forward, we must be rid of the stereotypes and labels and band together to achieve our greatest potentials (p. 195). Giese also mentions that to help this process go along quicker, boys should be taught and encouraged the same way girls are now taught and encouraged (p. 196).

Giese's intended general audience is anyone that has a close relationship with a young boy. This may include parents, brothers, sisters, and relatives. This book would also be quite valuable for educators, social workers, advocates, and post-secondary students looking into boy childhood and factors that shape it. Most importantly, the primary readership for this book is boys themselves. Being a boy that has gone through adolescence, I could relate to many of the points brought up, such as poor sexual education.

Overall, the book was very well written, backed up by facts and case studies. My criticism lies in the line "I suspect anyone who has a son in any sport has a story like this," which comes after describing the foul or aggressive language used by some parents to children. Her experiences were centered around hockey as the main sport which is popular for its aggression. The problem is that this experience cannot be generalized for every sport because many sports are low contact and very unaggressive, like track and field or swimming. I did track and field and swimming when I was younger, and I did not experience any aggressive behaviours from parents or fellow participants. However, Giese's use of her son's experience made the book more relevant and intimate, which shows that she is a mother that cares and wants others to know the reality of boys. 